\title{
Impact of an Onsite Endobronchial Ultrasound Program on the Time to Treatment of Cancer in Veterans
}

\author{
Van K Holden, MD. ${ }^{1,2}$, Stephanie Wappel, MD. ${ }^{1,2}$, Avelino C Verceles, MD, MS ${ }^{2}$ and Janaki Deepak MBBS ${ }^{1,2^{*}}$ \\ ${ }^{1}$ VA Maryland Health Care System, Baltimore VA Medical Center, USA \\ ${ }^{2}$ Division of Pulmonary \& Critical Care Medicine, Department of Medicine, University of Maryland School of Medicine, USA
}

\begin{abstract}
Background: Endobronchial ultrasound (EBUS) is commonly employed in the diagnostic evaluation of pulmonary nodules and for mediastinal staging of lung cancer. We hypothesized that the establishment of an onsite EBUS program would decrease the time to the diagnosis and treatment of detected malignancy in veterans seen in a dedicated pulmonary nodule clinic.
\end{abstract}

Methods: We conducted a retrospective chart review of patients seen at the Baltimore Veterans Affairs Medical Center (BVAMC) pulmonary nodule clinic and required diagnostic evaluation. One hundred and fifty consecutive patients before and after implementation of an onsite EBUS program were screened.

Results: Forty-nine patients from the pre-intervention group and 41 patients from the post-intervention group underwent diagnostic evaluation and were included in study analyses. There was no significant difference in the median number of days between CT chest and initial visit to the BVAMC Lung Mass Clinic ( 22 vs. 14 days, P = 0.06), CT chest to diagnostic procedure ( 47.5 vs. 37 days, $P=0.33$ ), and CT chest to initial treatment of detected malignancy (103 vs. 88 days, $P=0.15)$. There was no significant difference in the lung cancer stages or 1-year mortality between groups. A total of 76 barriers to care were identified among the 90 patients.

Conclusions: There was a trend towards improving the time from the initial CT chest to the treatment of cancer with onsite availability of EBUS. More efforts need to be done to decrease the modifiable barriers to treatment to improve the overall lung cancer care for veterans.

\section{Keywords}

Bronchoscopy, Endobronchial ultrasound, Lung cancer, Lymphadenopathy

\section{Abbreviations}

BVAMC: Baltimore Veterans Affairs Medical Center; EBUS: Endobronchial Ultrasound; UMMC: University of Maryland Medical Center

\section{Introduction}

Lung cancer is the leading cause of malignancy-related mortality in the U.S [1]. The National Lung Screening Trial demonstrated a $20 \%$ relative reduction in mortality from lung cancer with annual screening low-dose CT scans [2]. The annual rate of chest $\mathrm{CT}$ scanning and the percentage of positive scans with a lung nodule have already been increasing since 2006 [3] and will likely continue to increase with the implementation of lung cancer screening. Recommendations regarding the evaluation and management of pulmonary nodules include assessing the probability of malignancy, weighing the risks and benefits of different management strategies (biopsy, surgical resection, and surveillance with serial CT imaging), and shared decision-making [4]. Endobronchial ultrasound (EBUS) is commonly employed in the diagnostic evaluation of pulmonary nodules and for mediastinal staging.
The overall 5-year lung cancer survival rate is $17.8 \%$ but increases to $54 \%$ when the disease is still localized. Nevertheless, only $15 \%$ of lung cancer cases are diagnosed

\footnotetext{
*Corresponding author: Janaki Deepak, MBBS, Division of Pulmonary \& Critical Care Medicine, University of Maryland School of Medicine, $110 \mathrm{~S}$. Paca St., 2nd Floor, Baltimore, MD 21201, USA; VA Maryland Health Care System, Baltimore VA Medical Center, 10 N. Greene St., Baltimore, MD, 21201, USA, Tel: 410-328-8141, Fax: 410-328-0177

Accepted: June 12, 2019

Published online: June 14, 2019

Citation: Holden VK, Wappel S, Verceles AC, et al. (2019) Impact of an Onsite Endobronchial Ultrasound Program on the Time to Treatment of Cancer in Veterans. Ann Lung Cancer 3(1):66-74
} 
at an early stage [5]. A study in the United Kingdom showed a median time from first symptom onset to diagnosis of lung cancer of 91 days [6]. Other studies have shown a median symptom-to-treatment time interval ranging from 57 to 185 days [7-12]. These timeframes remain outside of Swedish, Canadian, and British Thoracic Society recommendations $[7,13,14]$. Thus, there is a need for significant improvement in the diagnosis and treatment timeframe of lung cancer.

Patients at the Baltimore Veterans Affairs Medical Center (BVAMC) who are found to have a pulmonary nodule(s) or mass with or without symptoms are referred to the Lung Mass Clinic. All referrals are screened by one dedicated pulmonologist with appointments made for selected patients with potentially higher risk lesions, as characterized by diameter $\geq 8 \mathrm{~mm}$, spiculated appearance, or upper lobe location. An EBUS program was established onsite at the BVAMC in early 2015. Prior to that, patients who required advanced diagnostic procedures were referred to the University of Maryland Medical Center (UMMC). All advanced bronchoscopic procedures (EBUS) at the BVAMC and UMMC were performed by the same two interventional pulmonologists. We aimed to characterize the diagnosis and treatment timeline of patients seen in this dedicated pulmonary nodule clinic. We hypothesized that the establishment of an onsite EBUS program at the BVAMC would decrease the time to the diagnosis and treatment of detected malignancy.

\section{Methods}

\section{Selection criteria}

We conducted a retrospective medical chart review of patients seen at the BVAMC Lung Mass Clinic. The medical records of 150 consecutive patients seen in clinic starting January 2013 (pre-intervention) and another 150 consecutive patients seen in clinic starting June 2015 (post-intervention) were reviewed. Radial and linear EBUS were made available onsite at the BVAMC in March 2015. This study was approved by the BVAMC and UMMC Institutional Review Boards. Written informed consent from study participants was not required.

Patients were included if they were at least 18 years of age without a prior history of lung cancer, and they were seen for an initial visit to the BVAMC Lung Mass Clinic for a new pulmonary nodule(s) or a known pulmonary nodule(s) not previously biopsied and subsequently underwent diagnostic evaluation. Exclusion criteria included insufficient information to characterize the appropriateness of nodule evaluation (i.e., death or loss to follow-up), reference to work-up performed outside the Veterans Affairs system without further details, or no pulmonary nodule size recorded. Patients with a benign pattern of calcification or innumerable nodules without a dominant nodule on CT imaging were also excluded. Other exclusion criteria included a known patient to the clinic presenting for follow-up; lack of pulmonary nodule, hilar mass or bulky lymphadenopathy on imaging; history of previous biopsy; prior history of lung cancer; or documented stability for at least 2 years of a known nodule.

\section{Data extraction}

Patient and imaging characteristics were obtained from chart review. Smoking status was determined as "neversmoker" if use was < 100 lifetime cigarettes. Symptoms suggestive of lung cancer as a reason for obtaining the CT chest was defined as hemoptysis, voice hoarseness, chest or shoulder pain, unexplained weight loss, shortness of breath or cough attributed to the lung nodule/mass, fatigue, or loss of appetite. In cases of multiple pulmonary nodules, the size of the dominant lesion was extracted from imaging reports for analyses. Results of PET/CT scans were considered positive if the report included concern for malignancy or SUV $\geq 2.5$. Malignancy probability was determined for pulmonary nodules based on the Mayo Clinic model [15]. Procedural and diagnostic outcomes were also collected. Timeframes from the initial CT chest to dates of the BVAMC clinic visit, diagnostic procedure, and initial treatment of cancer were calculated. Data on barriers to care were obtained and categorized as patient or system factors. The practice in the BVAMC is for all patients to undergo mediastinal staging using EBUS even if they do not have central lesions.

\section{Statistical analysis}

Thirty charts from the pre-intervention group were independently reviewed by two of the authors (V.H. and S.W.) to determine the intraclass correlation coefficient for the determination of timeframes. A high degree of reliability was found for timeframes between CT chest to initial clinic visit, diagnostic procedure, and treatment of lung cancer with intraclass correlation coefficients of 0.940, 0.999, and 0.999, respectively. Descriptive statistics were used to present the data. Timeframes were compared using medians and Wilcoxon rank sum test. Other continuous variables were summarized as means and standard deviations; categorical variables, as proportions. Paired t-test with a 2-sided distribution was used for comparisons of continuous variables, and chisquared test, for categorical variables. We defined statistical significance as a two-sided $P<0.05$. Statistical analyses were performed using SAS 9.4 (Cary, S.C, USA).

\section{Results}

Forty-nine patients from the pre-intervention group $(1 / 2013$ - 10/2013) and 41 patients from the post-intervention group (6/2015 - 2/2016) underwent diagnostic evaluation and were included in study analyses (Figure 1). There was no statistically significant difference in baseline characteristics between the two groups, including age, sex, smoking history and $\mathrm{FEV}_{1}$ (Table 1). Combined, the cohort were all men with a mean age of 66 years, 50 pack-year smoking history, and $\mathrm{FEV}_{1} 2.2 \mathrm{~L}$.

There were a variety of indications for the CT chest, including symptoms suggestive of lung cancer, symptoms not likely related to the nodule, lung cancer screening, follow-up of a known pulmonary nodule, and other (Table 1). There was a significant difference in the reason for the $\mathrm{CT}$ chest between the two groups, with a decrease in the number of patients with symptoms not likely related to the nodule in the post-intervention group. There was no difference in the mean nodule diameter between groups (28.6 vs. $29.9 \mathrm{~mm}$, 


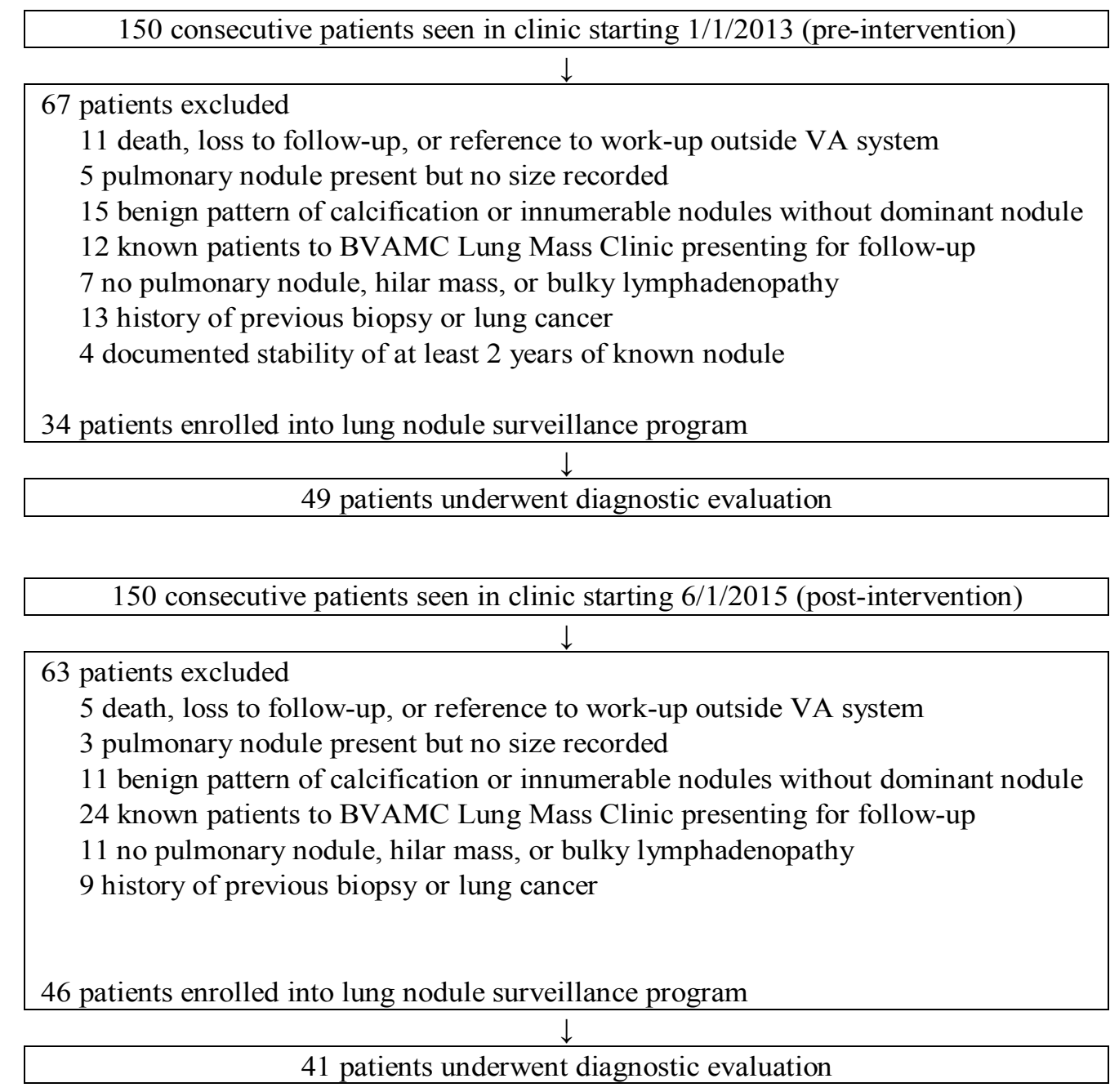

Figure 1: Patient selection.

Table 1: Patient and imaging characteristics.

\begin{tabular}{|l|l|l|}
\hline Attribute & Pre-, N (\%) & Post-, N (\%) \\
\hline Patients & 49 & 41 \\
\hline Age, years (mean) & $65.5 \pm 10$ & $67.4 \pm 7.2$ \\
\hline Male sex & 49 & 41 \\
\hline Smoking history & & \\
\hline Never smokers & $5(10.2)$ & 0 \\
\hline Former smokers & $18(36.7)$ & $19(46.3)$ \\
\hline Current smokers & $26(53.1)$ & $22(53.7)$ \\
\hline Pack-years (mean) & $52.6 \pm 26.9$ & $47.4 \pm 31.7$ \\
\hline Family history of lung cancer & $12(24.5)$ & $9(22.0)$ \\
\hline FEV1, Liters (mean) & $2.09 \pm 0.74$ & $2.24 \pm 0.72$ \\
\hline Reason for CT chest & & 0.10 \\
\hline Symptoms suggestive of lung cancer & $17(34.7)$ & $11(26.8)$ \\
\hline Symptoms not likely related to the nodule & $23(46.9)$ & $6(14.6)$ \\
\hline Lung cancer screening & 0 & $3(7.3)$ \\
\hline Follow-up of known pulmonary nodule & $5(10.2)$ & $4(9.8)$ \\
\hline Other & $4(8.2)$ & $17(41.5)$ \\
\hline
\end{tabular}


Citation: Holden VK, Wappel S, Verceles AC, et al. (2019) Impact of an Onsite Endobronchial Ultrasound Program on the Time to Treatment of Cancer in Veterans. Ann Lung Cancer 3(1):66-74

\begin{tabular}{|c|c|c|c|}
\hline Nodule size, mm (mean) & $28.6 \pm 22.6$ & $29.9 \pm 20.6$ & 0.80 \\
\hline Upper lobe nodule location & $28(62.2)$ & $20(60.6)$ & 0.88 \\
\hline \multicolumn{3}{|l|}{ Nodule characteristics } & \multirow[t]{5}{*}{0.12} \\
\hline Solid, not spiculated & $25(55.6)$ & $16(48.5)$ & \\
\hline Solid, spiculated/lobulated & $20(44.4)$ & $13(39.4)$ & \\
\hline Semisolid & 0 & $1(3.0)$ & \\
\hline Pure ground-glass opacity & 0 & $3(9.1)$ & \\
\hline Presence of emphysema & $31(63.3)$ & $26(63.4)$ & 0.99 \\
\hline Presence of bulky lymphadenopathy or hilar mass & $24(49.0)$ & $21(51.2)$ & 0.83 \\
\hline \multicolumn{3}{|l|}{$\mathrm{PET} / \mathrm{CT}$ result } & \multirow[t]{4}{*}{0.54} \\
\hline Not performed & $9(18.4)$ & $8(19.5)$ & \\
\hline Negative & 0 & $1(2.4)$ & \\
\hline Positive & $40(81.6)$ & $32(78.1)$ & \\
\hline
\end{tabular}

${ }^{*} P$-value reached significance at $<0.05$.

Table 2: Procedural and diagnostic characteristics.

\begin{tabular}{|c|c|c|c|}
\hline Attribute & Pre-, N (\%) & Post-, N (\%) & $P$ value \\
\hline Patients & 49 & 41 & \\
\hline \multicolumn{3}{|l|}{ Malignancy probability } & \multirow[t]{3}{*}{0.40} \\
\hline Intermediate, 5-65\% & $20(44.4)$ & $9(29.0)$ & \\
\hline High, > 65\% & $24(53.3)$ & $21(67.7)$ & \\
\hline \multicolumn{3}{|l|}{ Diagnostic procedure } & \multirow[t]{7}{*}{$0.006^{*}$} \\
\hline Lobectomy or wedge resection & $1(2.0)$ & $3(7.3)$ & \\
\hline Transthoracic needle aspiration & $11(22.5)$ & 0 & \\
\hline Bronchoscopy & $3(6.1)$ & 0 & \\
\hline EBUS or navigational bronchoscopy & $30(61.2)$ & $33(80.5)$ & \\
\hline Mediastinoscopy & 0 & 0 & \\
\hline Other procedure & $4(8.2)$ & $5(12.2)$ & \\
\hline Initial procedure provided diagnosis ${ }^{\dagger}$ & $39(79.6)$ & $38(92.7)$ & 0.08 \\
\hline \multicolumn{3}{|l|}{ Histological diagnosis } & \multirow[t]{7}{*}{0.58} \\
\hline Lung adenocarcinoma & $13(27.1)$ & $13(31.7)$ & \\
\hline Squamous cell carcinoma & $12(25.0)$ & $14(34.2)$ & \\
\hline Small cell carcinoma & $5(10.4)$ & $2(4.9)$ & \\
\hline Other NSCLC & $2(4.2)$ & $3(7.3)$ & \\
\hline Other malignancy & $6(12.5)$ & $5(12.2)$ & \\
\hline Benign & $10(20.8)$ & $4(9.8)$ & \\
\hline \multicolumn{3}{|l|}{ Lung cancer stage } & \multirow[t]{5}{*}{0.69} \\
\hline 1 & $9(28.1)$ & $12(35.3)$ & \\
\hline II & $3(9.4)$ & $5(14.7)$ & \\
\hline III & $9(28.1)$ & $6(17.7)$ & \\
\hline IV & $11(34.4)$ & $11(32.4)$ & \\
\hline 1-year mortality & $10(20.4)$ & $14(34.2)$ & 0.14 \\
\hline
\end{tabular}

Definition of abbreviations: EBUS: Endobronchial Ultrasound; NSCLC: Non-Small Cell Lung Cancer. ${ }^{*} P$-value reached significance at $<0.05$. tSix patients in the pre-intervention group and two in the post-intervention group required additional diagnostic procedures.

$P=0.80$ ), or in PET/CT results, with $81.6 \%$ vs. $78.1 \%$ having a positive result and a similar number of patients who did not undergo PET/CT scan $(P=0.54)$. The majority of the nodules were located in the upper lobes with solid characteristics in both groups. Almost two-thirds of patients from each group had evidence of emphysema on imaging, and half of patients 


\section{Lung Cancer Stage}

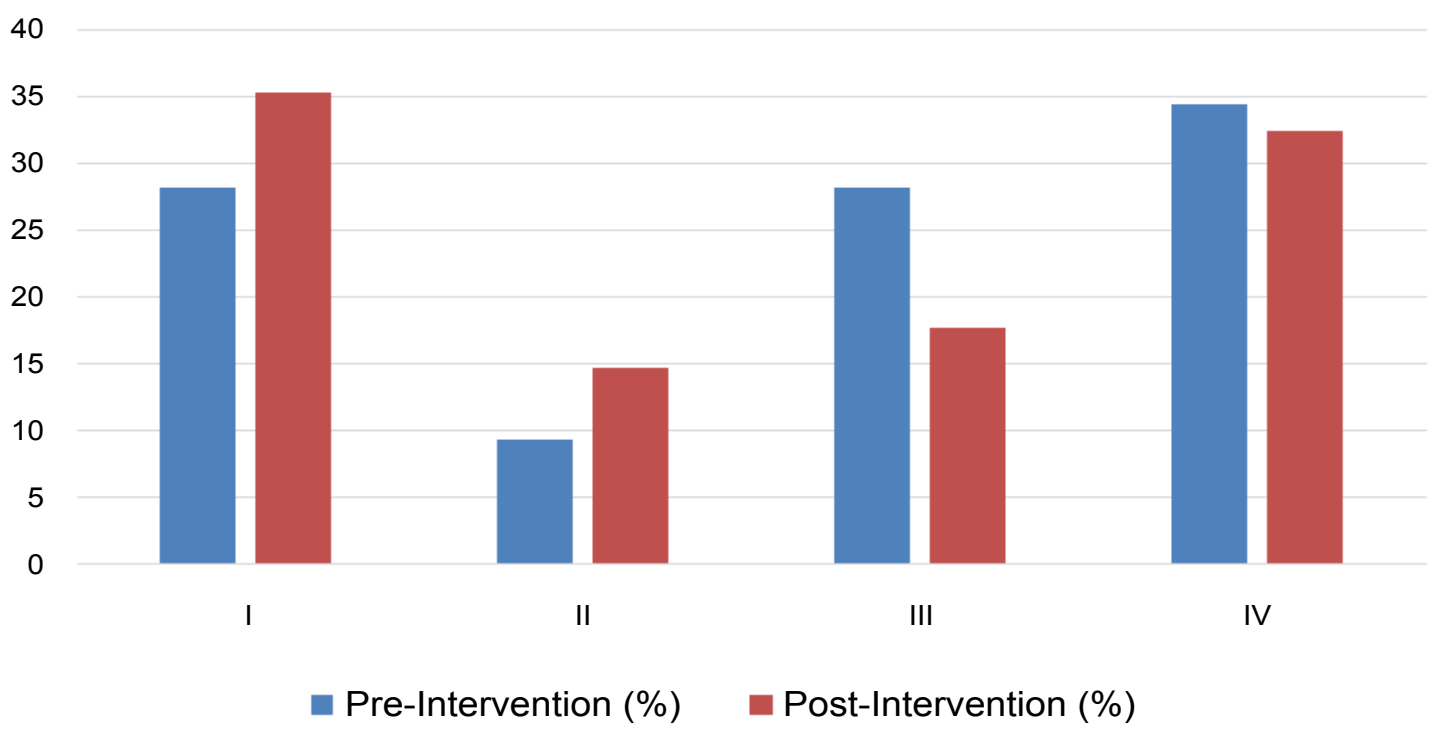

Figure 2: Comparison of lung cancer stages in the pre-intervention and post-intervention groups.

had the presence of bulky lymphadenopathy or hilar mass.

The majority of patients had a high probability of malignancy $(53.5 \%$ vs. $67.7 \%)$, and there was no difference in malignancy probability between the two groups (Table 2). There was a significant difference in the type of diagnostic procedures undertaken in the post-intervention group. The number of transthoracic needle aspirations decreased from $22.5 \%$ to $0 \%$, and flexible bronchoscopies, $6.1 \%$ to $0 \%$ after the implementation of an onsite EBUS program. However, the rate of EBUS or navigational bronchoscopy increased from $61.2 \%$ to $80.5 \%$. There was also an increase in the rate of lobectomies or wedge resections as the initial diagnostic procedure, $2.0 \%$ vs. $7.3 \%$. None of the patients underwent mediastinoscopy. There was no significant difference in the diagnostic yield of the initial procedure, $79.6 \%$ vs. $92.7 \%(P=0.08)$.

The most commonly identified malignancies were lung adenocarcinoma and squamous cell carcinoma with no significant difference between groups in rates of other histological diagnoses. There was no significant difference in the lung cancer stages (Figure 2 ) or 1-year mortality rate $(20.4 \%$ vs. $34.2 \%$, $\mathrm{P}=0.14$ ) between groups.

There was no significant difference in the median number of days between CT chest and initial visit to the BVAMC Lung Mass Clinic (22 vs. 14 days, $P=0.06$ ), CT chest to diagnostic procedure ( 47.5 vs. 37 days, $P=0.33$ ), and CT chest to initial treatment of detected malignancy (103 vs. 88 days, $P=0.15$ ) (Figure 3 ). The combined cohort had an overall median of 18 days between $\mathrm{CT}$ chest and clinic visit with a specialist ("referral delay"), 18 days between that visit and diagnosis ("specialist's delay"), and 38 days between diagnosis and treatment ("treatment delay") (Figure 4). Ten of 37 patients $(27.0 \%)$ received cancer treatment within 60 days of their CT chest in the pre-intervention group compared to $12 / 34$ $(35.3 \%)$ in the post-intervention group.
A total of 76 barriers to care were identified among the 90 patients. The leading barrier to care ( $n=28$ or $36.8 \%$ ) was comorbidities that delayed the diagnostic procedure or initiation of treatment. Other patient-related barriers to care included missed appointments ( $n=17$ or $22.4 \%$ ) (Mainly related to lack of understanding about the disease process) and declining the diagnostic procedure or treatment $(n=11$ or $14.5 \%$ ). The most common system-related barrier was non-availability of specific treatment, such as stereotactic radiation therapy ( $n=8$ or $10.5 \%$ ).

\section{Discussion}

Our study found no significant difference in the timeframes from the initial CT chest to the diagnosis and treatment of identified malignancy with the implementation of an onsite EBUS program. The median time from CT chest to the treatment of cancer was 88 days in the post-intervention group. Although this is comparable to previously published studies, it remains outside of recommended guidelines. In addition, despite the lack of statistical significance, the difference of 15 days between the two groups may represent a clinically significant time period with added stress and anxiety for the patient.

Studies have demonstrated a median symptom-to-treatment time interval ranging from 57 to 185 days [7-12]. These timeframes remain outside of Swedish, Canadian, and British recommendations. The Swedish Lung Cancer Study Group recommends that $80 \%$ of all patients' diagnostic tests should be completed within 4 weeks from consultation with a specialist and that treatment should start within 2 weeks thereafter [7]. In Canadian recommendations, a maximum of 4 weeks should elapse between the first visit to a general practitioner and diagnosis [13]. British Thoracic Society guidelines recommend that diagnostic results should be communicated to the patient within 2 weeks of deciding to perform a pro- 

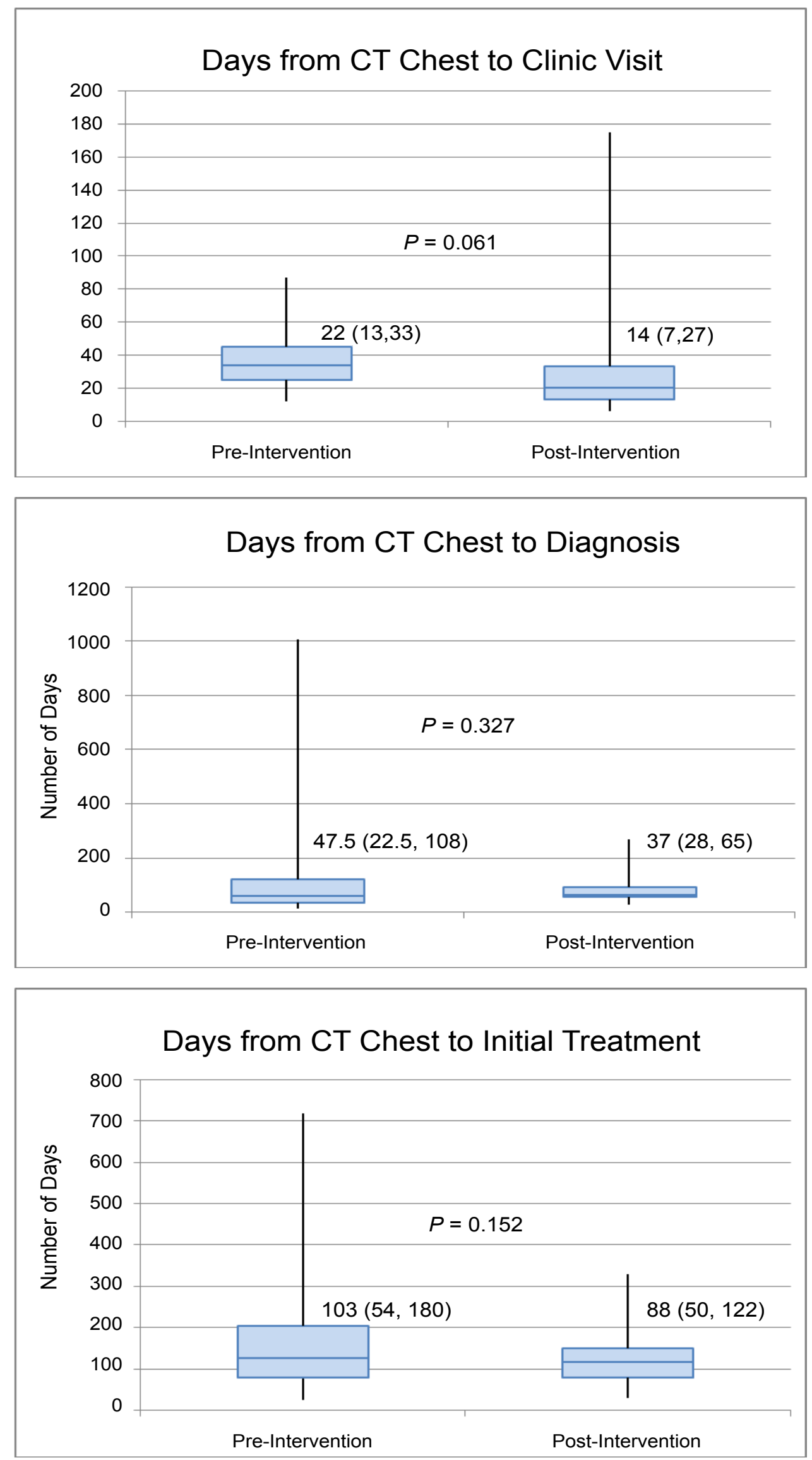

Figure 3: Comparison of timeframes from the initial CT chest to initial visit to the BVAMC Lung Mass Clinic, diagnostic procedure, and initial treatment of detected malignancy. Values are listed as medians with interquartile ranges. 


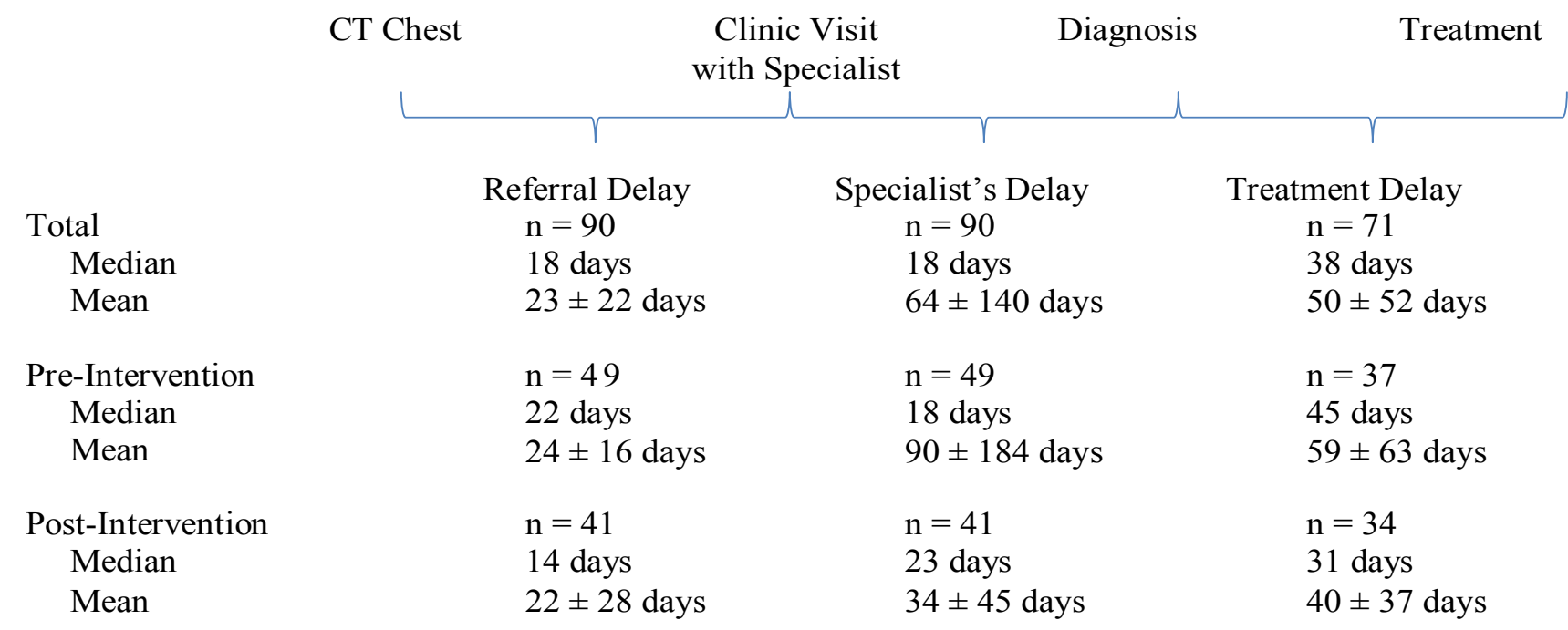

Figure 4: Time intervals between CT chest, clinic visit with specialist, diagnosis, and treatment of detected malignancy. Values are listed as medians and means with standard deviations.

cedure. It also endorses a maximum of 8 weeks between the first specialist consultation and thoracotomy [14].

Treatment timeframes have been evaluated based on intervals between symptom onset to first visit with a physician (patient's delay) to first visit with a specialist (referral delay) to diagnosis (specialist's delay) and finally treatment (treatment delay) [8]. Our study demonstrated no significant difference between groups in the time from initial CT chest to diagnosis. This suggests that system processes were already in place to provide timely access to diagnostic interventions prior to the availability of onsite EBUS at the BVAMC. However, the overall median of 18 days for the specialist's delay is still outside of British Thoracic Society guidelines. Previous studies have shown a median of 9-15 days for this time interval $[8,9,16]$. The treatment delay was a median of 38 days overall in our study, with a difference of 14 days between groups. This suggests that cancer care may be expedited when the diagnostic evaluation is performed onsite, likely due to the accessibility of procedural and pathology reports within the institution. Overall, both the diagnostic and treatment processes for cancer care could be improved.

Previous studies have demonstrated mixed results on the effect of treatment delays on prognosis. A shorter delay was associated with a poorer prognosis due to an expedited diagnostic process in advanced patients [7-9], who may present with neurological symptoms, appetite loss, hemoptysis, or pain [16]. Patients with older age and multiple comorbidities $[16,17]$ or who initially declined bronchoscopy or underwent numerous diagnostic procedures [8] had a longer delay to initiating treatment. Definitive surgical resection for early-stage lung cancer performed 38 days or more after diagnosis had significantly worse 5-year survival [18].

There was a high rate of comorbidities in our veteran population, which precluded procedures and stage specific treatment options. In addition, a high number of patients declined an intervention or missed appointments. This is possibly due to the lack of understanding and education about the significance of these procedures. The knowledge deficit surrounding pulmonary nodules can be associated with severe distress in veterans $[19,20]$. Many of the multilevel barriers to effective lung cancer screening that have been described are also applicable to the evaluation of detected pulmonary nodules. Patient-level barriers include fear, lack of awareness, limited access to care due to financial or social factors, and mistrust of the healthcare system. System-level barriers include limited resources, competing demands for those resources, and complexity of implementation [21].

Our results suggest that veterans are a population at highrisk for lung cancer as $27-32 \%$ of patients seen in the BVAMC Lung Mass Clinic subsequently underwent diagnostic evaluation. In addition, their baseline characteristics are in-line with those recommended for lung cancer screening [2]; however, only 3 out of the entire cohort had a CT chest for lung cancer screening. The lack of an established lung cancer screening program at BVAMC meant that mainly symptomatic patients with higher stage and increased disease burden were brought to our notice. This is exemplified by the fact that by the time nodules are seen for diagnostic evaluation, they are sizable at $2.9 \mathrm{~cm}$ with high-risk characteristics (upper lobe; solid; emphysema) and high malignancy probability. In addition, half of patients already had associated bulky lymphadenopathy or hilar mass. Despite this, $18-19 \%$ of patients did not undergo $\mathrm{PET} / \mathrm{CT}$ scan. Studies have demonstrated inconsistent rates of compliance with guidelines, including one study performed in Veterans Affairs facilities which showed that $44.7 \%$ of patients with a detected nodule received care inconsistent with guidelines [22-24].

Our study found no statistical significance in stage shift or 1-year mortality between groups; however, there were other advantages identified with the initiation of an onsite EBUS program. We did detect a higher rate of lung cancers diagnosed as stage $\mathrm{I} / \mathrm{II}$ with a slight decrease in diagnoses of 
stage III/IV after the availability of onsite EBUS. The percentage of lung cancers stage $\mathrm{I} / \mathrm{II}$ and IV is similar to a previous study that demonstrated rates of $37 \%$ and $33 \%$, respectively, prior to the implementation of lung cancer screening in a veteran population [25]. Infrastructure requirements for the establishment of a lung cancer screening program include the ability to characterize nodules with nonsurgical approaches, including bronchoscopic biopsy, and track outcomes of testing (complications and diagnosis) [26-28]. Thus, an onsite EBUS program facilitates the future establishment of a lung cancer screening program but may not necessarily expedite the time from detection to diagnosis. After implementation of lung cancer screening programs at 8 academic Veterans Health Administration hospitals, 1.5\% (31/2106) of screened patients was confirmed to have lung cancer with a mean of 137 days (range 5-330) from initial CT scan to diagnosis [29]. This is in contrast to our median of 37 days from initial CT scan to diagnosis without a lung cancer screening program as patients in our cohort presented with symptoms and higher disease burden, requiring more urgent evaluation.

An onsite EBUS program decreased the need for transthoracic needle aspiration and flexible bronchoscopy with conventional transbronchial needle aspiration, with a trend toward increasing the diagnostic yield of the initial procedure post-intervention. This decreased the need for a second diagnostic procedure and likely expedited the time to treatment. One study demonstrated a decreased time from symptom to diagnosis with lymph node fine needle aspiration or biopsy compared to other procedures, such as CT-guided biopsy, pleural fluid cytology or biopsy, and sputum cytology [10].

There are some limitations to this study. We grouped EBUS and navigational bronchoscopy as a single diagnostic procedure category; although, navigational bronchoscopy was not available at the VA. However, we wanted to be broad in order to assess the impact of implementation of just an onsite EBUS program. In addition, the numbers in this study are small and probably understate the actual significance of having an onsite EBUS program. The follow-up period was only one year. Finally, the findings in our study may not be applicable to non-Veterans Health Administration hospitals and non-veterans. This lack of understanding of disease process and the imperativeness of obtaining the diagnosis in a time sensitive manner were also significant limiting factors.

\section{Conclusions}

In conclusion, our study shows a trend towards improving the time from the initial CT chest to the treatment of cancer with onsite availability of EBUS, which is the standard of care for lung cancer diagnosis and mediastinal staging. More efforts need to be done to decrease the modifiable barriers to treatment to improve the overall lung cancer care for our veterans.

\section{Acknowledgements}

Edward Pickering, MD, interventional pulmonologist and Ashutosh Sachdeva, MBBS, interventional pulmonologist.

\section{Author Contributions}

All authors contributed to study conception and design, interpretation of data, drafting of the manuscript for important intellectual content, and final approval of the version to be published. Statistical analysis of data: VH, AV.

\section{Conflicts of Interest and Source of Funding}

JD has funding from the following source "National Institutes of Health, Laboratory of Human Carcinogenesis, CCR, NCl, NO2RC-57700. Resource collection and evaluation of human tissues and cells from donors with an epidemiology profile".

\section{Disclaimer}

This material is the result of work supported with resources and the use of facilities at the VA Maryland Health Care System, Baltimore, Maryland. The contents do not represent the views of the U.S. Department of Veterans Affairs or the United States Government.

\section{Funding}

This research did not receive any specific grant from funding agencies in the public, commercial, or not-for-profit sectors.

\section{References}

1. U.S. Cancer Statistics Working Group (2015) United States cancer statistics: 1999-2012 incidence and mortality web-based report. Department of Health and Human Services, Centers for Disease Control and Prevention, and National Cancer Institute, Atlanta, GA.

2. Aberle DR, Adams AM, Berg CD, et al. (2011) Reduced lung-cancer mortality with low-dose computed tomographic screening. $\mathrm{N}$ Engl J Med 365: 395-409.

3. Gould MK, Tang T, Liu IL, et al. (2015) Recent trends in the identification of incidental pulmonary nodules. Am J Respir Crit Care Med 192: 1208-1214.

4. Gould MK, Donington J, Lynch WR, et al. (2013) Evaluation of individuals with pulmonary nodules: When is it lung cancer? Diagnosis and management of lung cancer, (3rd edn): American College of Chest Physicians evidence-based clinical practice guidelines. Chest 143: e93S-e120S.

5. U.S. National Institutes of Health. SEER Cancer Statistics Review, 1975-2011. National Cancer Institute.

6. Walter FM, Rubin G, Bankhead C, et al. (2015) Symptoms and other factors associated with time to diagnosis and stage of lung cancer: A prospective cohort study. Br J Cancer 112: S6-S13.

7. Myrdal G, Lambe M, Hillerdal G, et al. (2004) Effect of delays on prognosis in patients with non-small cell lung cancer. Thorax 59: 45-49.

8. Salomaa ER, Sallinen S, Hiekkanen H, et al. (2005) Delays in the diagnosis and treatment of lung cancer. Chest 128: 2282-2288.

9. Annakkaya AN, Arbak P, Balbay O, et al. (2007) Effect of symptom-to-treatment interval on prognosis in lung cancer. Tumori 93: 61-67.

10. Chandra S, Mohan A, Guleria R, et al. (2009) Delays during the diagnostic evaluation and treatment of lung cancer. Asian Pacific J Cancer Prev 10: 453-456. 
Citation: Holden VK, Wappel S, Verceles AC, et al. (2019) Impact of an Onsite Endobronchial Ultrasound Program on the Time to Treatment of Cancer in Veterans. Ann Lung Cancer 3(1):66-74

11. Billing JS, Wells FC (1996) Delays in the diagnosis and surgical treatment of lung cancer. Thorax 51: 903-906.

12. Yaman N, Ozgen A, Celik P, et al. (2009) Factors affecting the interval from diagnosis to treatment in patients with lung cancer. Tumori 95: 702-705.

13. Simunovic M, Gagliardi A, McCready D, et al. (2001) A snapshot of waiting times for cancer surgery provided by surgeons affiliated with regional cancer centres in Ontario. Can Med Assoc J 165: 421-425.

14. British Thoracic Society (1998) BTS recommendations to respiratory physicians for organizing the care of patients with lung cancer: The lung cancer working party of the british thoracic society standards of care committee. Thorax 53: S1-S8.

15. Swensen SJ, Silverstein MD, Ilstrup DM, et al. (1997) The probability of malignancy in solitary pulmonary nodules. Application to small radiologically indeterminate nodules. Arch Intern Med 157: 849-855.

16. Lovgren $M$, Levealahti $H$, Tishelman $C$, et al. (2008) Time spans from first symptom to treatment in patients with lung cancerthe influence of symptoms and demographic characteristics. Acta Oncol 47: 397-405.

17. Kudjawu YC, Chatellier G, Decool E, et al. (2016) Timing in initiating lung cancer treatment after bronchoscopy in France: Study from medico-administrative database. Lung Cancer 95: 44-50.

18. Yang CJ, Wang H, Kumar A, et al. (2017) Impact of timing of lobectomy on survival for clinical stage IA lung squamous cell carcinoma. Chest 152: 1239-1250.

19. Slatore CG, Golden SE, Ganzini L, et al. (2015) Distress and patientcentered communication among veterans with incidental (not screen-detected) pulmonary nodules. A cohort study. Ann Am Thorac Soc 12: 184-192.

20. Slatore CG, Press N, Au DH, et al. (2013) What the heck is a "nodule"? A qualitative study of veterans with pulmonary nodules. Ann Am Thorac Soc 10: 330-335.

21. Carter-Harris L, Gould MK (2017) Multilevel barriers to the successful implementation of lung cancer screening. Why does it have to be so hard? Ann Am Thorac Soc 14: 1261-1265.

22. Tanner NT, Aggarwal J, Gould MK, et al. (2015) Management of pulmonary nodules by community pulmonologists: A multicenter observational study. Chest 148: 1405-1414.

23. Wiener RS, Gould MK, Slatore CG, et al. (2014) Resource use and guideline concordance in evaluation of pulmonary nodules for cancer: too much and too little care. JAMA Intern Med 174: 871880.

24. Veeramachaneni NK, Crabtree TD, Kreisel D, et al. (2009) A thoracic surgery clinic dedicated to indeterminate pulmonary nodules: Too many scans and too little pathology? J Thorac Cardiovasc Surg 137: 30-35.

25. Okereke IC, Bates MF, Jankowich MD, et al. (2016) Effects of implementation of lung cancer screening at one veterans affairs medical center. Chest 150: 1023-1029.

26. Mazzone P, Powell CA, Arenberg D, et al. (2015) Components necessary for high-quality lung cancer screening: American college of chest physicians and american thoracic society policy statement. Chest 147: 295-303.

27. Wiener RS, Gould MK, Arenberg DA, et al. (2015) An official American Thoracic Society/American College of Chest Physicians policy statement: Implementation of low-dose computed tomography lung cancer screening programs in clinical practice. Am J Respir Crit Care Med 192: 881-891.

28. Tukey MH, Clark JA, Bolton R, et al. (2016) Readiness for implementation of lung cancer screening. A national survey of veterans affairs pulmonologists. Ann Am Thorac Soc 13: 1794-1801.

29. Kinsinger LS, Anderson C, Kim J, et al. (2017) Implementation of lung cancer screening in the veterans health administration. JAMA Intern Med 177: 399-406

DOI: $10.36959 / 825 / 581$

Copyright: (c) 2019 Holden VK, et al. This is an open-access article distributed under the terms of the Creative Commons Attribution License, which permits unrestricted use, distribution, and reproduction in any medium, provided the original author and source are credited. 